\title{
Article \\ Role of Single Nucleotide Variants in FSHR, GNRHR, ESR2 and LHCGR Genes in Adolescents with Polycystic Ovary Syndrome
}

\author{
Lasma Lidaka $^{1, *(0)}$, Laine Bekere ${ }^{2}\left({ }^{\circ}\right.$, Adele Rota ${ }^{3}$, Jekaterina Isakova ${ }^{3}$, Gunta Lazdane ${ }^{4}\left(\right.$, Anda Kivite-Urtane ${ }^{4}$, \\ Iveta Dzivite-Krisane ${ }^{5}$, Inga Kempa ${ }^{3}$, Zane Dobele ${ }^{3}$ and Linda Gailite ${ }^{3}$ (1) \\ 1 Department of Paediatric Gynaecology, Children's Clinical University Hospital, LV-1004 Riga, Latvia \\ 2 Department of Obstetrics and Gynaecology, Riga Stradins University, LV-1007 Riga, Latvia; \\ laine.bekere@gmail.com \\ 3 Scientific Laboratory of Molecular Genetics, Riga Stradins University, LV-1007 Riga, Latvia; \\ dr.adele.rota@gmail.com (A.R.); jekaterina.isakova.25@gmail.com (J.I.); inga.kempa@rsu.lv (I.K.); \\ zane.dobele@rsu.lv (Z.D.); linda.gailite@rsu.lv (L.G.) \\ 4 Institute of Public Health, Riga Stradins University, LV-1007 Riga, Latvia; gunta.lazdane@rsu.lv (G.L.); \\ anda.kivite-urtane@rsu.lv (A.K.-U.) \\ 5 Department of Paediatric Endocrinology, Children's Clinical University Hospital, LV-1004 Riga, Latvia; \\ dzivite@bkus.lv \\ * Correspondence: lasma.lidaka@bkus.lv
}

\section{check for} updates

Citation: Lidaka, L.; Bekere, L.; Rota, A.; Isakova, J.; Lazdane, G.;

Kivite-Urtane, A.; Dzivite-Krisane, I.; Kempa, I.; Dobele, Z.; Gailite, L. Role of Single Nucleotide Variants in FSHR, GNRHR, ESR2 and LHCGR Genes in Adolescents with Polycystic Ovary Syndrome. Diagnostics 2021, 11, 2327. https://doi.org/10.3390/ diagnostics11122327

Academic Editor: Edward J. Pavlik

Received: 30 October 2021

Accepted: 6 December 2021

Published: 11 December 2021

Publisher's Note: MDPI stays neutral with regard to jurisdictional claims in published maps and institutional affiliations.

Copyright: (c) 2021 by the authors. Licensee MDPI, Basel, Switzerland. This article is an open access article distributed under the terms and conditions of the Creative Commons Attribution (CC BY) license (https:// creativecommons.org/licenses/by/ $4.0 /)$.
Abstract: Background: Polycystic ovary syndrome (PCOS) is the most common endocrinopathy in women, affecting up to $16.6 \%$ of reproductive-age women. PCOS symptoms in adolescents comprise oligomenorrhoea/amenorrhoea and biochemical and/or clinical hyperandrogenism. Long-term health risks of PCOS patients include infertility, metabolic syndrome, type 2 diabetes and cardiovascular disease. Genetic factors have been proven to play a role in development of the syndrome and its symptoms. Objective: To investigate single nucleotide variants (SNVs) in the GNRHR, ESR2, LHCGR and FSHR genes in adolescent patients with PCOS and their association with PCOS symptoms. Methods: We conducted a cross-sectional study comprising of 152 adolescents: 63 patients with PCOS, 22 patients at risk of developing PCOS and 67 healthy controls. Participants were recruited from out-patients attending a gynaecologist at the Children's Clinical University Hospital, Riga, Latvia, between January 2017 and December 2020. Genomic DNA was extracted from whole blood, and SNVs in the GNRHR, ESR2, LHCGR and FSHR genes were genotyped. The distributions of SNV genotypes were compared among the three groups and genotype-phenotype associations within the PCOS group were evaluated. Results: No statistically significant differences were found in the distributions of genotypes for GNRHR (rs104893837), ESR2 (rs4986938), LHCGR (rs2293275) and FSHR (rs6166, rs6165, rs2349415) among PCOS patients, risk patients and healthy controls. Within the PCOS group, ESR2 rs4986938 minor allele homozygous patients had a significantly higher level of total testosterone than major allele homozygous patients and heterozygous patients. A significantly higher total testosterone level was also observed in PCOS patients carrying the LHCGR rs2293275 minor allele compared with major allele homozygous patients. Conclusions: The SNVs ESR2 rs4986938 and LHCGR rs2293275 play a role in the phenotypic characteristics of PCOS. To fully uncover their influence on the development of PCOS and its symptoms, further studies of larger cohorts and a follow up of this study sample through to adulthood are required. Furthermore, studies of adolescent PCOS patients conducted prior to the latest European Society of Human Reproduction and Embryology (ESHRE) criteria (2018) should be re-evaluated as the study groups might include risk patients according to these updated criteria, thereby potentially significantly impacting the published results.

Keywords: adolescents; genetics; GNRHR; ESR2; LHCGR; FSHR; polycystic ovary syndrome 


\section{Introduction}

Polycystic ovary syndrome (PCOS) is the most common endocrinopathy in women, affecting more than $15 \%$ of reproductive-age women. In the adolescent population, it is characterised by oligo/anovulation and biochemical and/or clinical hyperandrogenism [1]. As PCOS most often presents during adolescence, it is commonly masked by symptoms of normal puberty, e.g., acne and menstrual irregularities. The main treatment to target pathophysiological mechanisms of PCOS and to improve later prognosis, is lifestyle changes. Therefore, a timely diagnosis via genetic testing is crucial to avoid long-term consequences of the syndrome [1].

Family clustering [2,3], genome-wide association studies (GWAS) [4] and research examining the role of environmental factors have established that PCOS is a multifactorial disease [5]. The genetics of PCOS appear to be very complex. Hypothalamichypophysis-ovarian (HHO) axis dysfunction is a pivotal mechanism in the development of the syndrome. Signal transduction in the main regulatory pathway is executed via the gonadotropin-releasing hormone receptor (GNRHR) and steroid hormone receptors. To date, 19 PCOS susceptibility loci have been identified in various GWAS, with four of them located near genes involved in the HHO axis [4]. It might be expected that these GWAS would provide a precise answer regarding the possible genetic cause of PCOS and genotype-phenotype interactions; however, their results are diverse [4]. An explanation for this disparity could be that the robust analyses used in GWAS may overlook genes that have a weak association with the syndrome and rare genetic variants that cause PCOS and influence its symptoms [4]. Therefore, at present, the GWAS available in the literature merely offer contradictory and inconclusive results regarding the association of different single nucleotide variants (SNVs) with the development of PCOS and its related symptoms.

To help address this discordance related to PCOS genetic association studies, we focused our research on SNVs in genes encoding proteins important in the $\mathrm{HHO}$ axis, namely GNRHR (rs104893837), oestrogen receptor 2 (ESR2 rs4986938), luteinising hormone/choriogonadotropin receptor (LHCGR rs2293275) and follicle-stimulating hormone receptor (FSHR rs6166, rs6165, rs2349415). The SNVs were selected based on previous GWAS or other relevant studies. In the case of GNRHR, it was selected because it was observed most frequently in the Latvian population [unpublished results]. To the best of our knowledge, only one study investigating genetic polymorphisms in adolescents with PCOS has been published to date. This study by Unsal et al. examined several genes, but only rs6166 and rs6165 SNVs in FSHR are in common with our selected SNVs [6].

\section{Materials and Methods}

\subsection{Study Population}

The study sample of this cross-sectional study consisted of 152 adolescents: 63 patients with PCOS, 22 patients at risk of developing PCOS (see below) and 67 healthy controls. All participants were of European ancestry and attended a single paediatric gynaecologist at the Children's Clinical University Hospital, Riga, Latvia, at least a year after menarche. The Children's Clinical University Hospital is the only specialized children's hospital in Latvia, providing a whole spectrum of services—both in-patient and out-patient—designed specifically for children up to 18 years of age. All services are financed by the state budget and gynaecologists are accessible without a referral from general practitioners or other specialists. This allows utilization of services by patients from different regions and socioeconomic backgrounds. There is no national registry for PCOS patients, but considering the facility for patient recruitment, we could be sure that the study sample consisted of a homogenous Caucasian population, encapsulating the whole population of the country. Recruitment took place between 1 January 2017 and 31 December 2020. A diagnosis of PCOS was established according to the European Society of Human Reproduction and Embryology (ESHRE) 2018 criteria: biochemical (defined as an elevated total testosterone (T) level above the normal laboratory range according to Tanner staging) and/or clinical hyperandrogenism (modified Ferriman-Gallwey $(\mathrm{mFG})$ score $\geq 4$ ) and oligomenorrhoea 
(defined as a menstrual cycle length more than 45 days at least a year after menarche or more than 35 days three years after menarche) [1]. Healthy adolescents who attended the same gynaecologist for other issues (such as contraception counselling, etc.) were included in the study as a control group. Additionally, patients with hirsutism but who did not fulfil all the PCOS criteria and did not have any exclusion criteria were included in the so-called 'risk' group [1].

Patients with other known gynaecological and endocrinological disorders (Cushing's syndrome, hypothyroidism, endocrine tumours, diabetes, etc.) or other serious comorbidities (e.g., severe liver or kidney disease) and patients who had used hormonal medication within the previous six months were excluded from the study. The study was approved by the Central Medical Ethics Committee of Latvia (protocol no: 1/16-04-12) and followed the guidelines of the Declaration of Helsinki. All participants gave their signed informed consent. Permission was also received from legal guardians for those participants under the age of 16 .

\subsection{Patient Examination}

Clinical examination of participants included body mass index (BMI), waist-hip ratio, mFG score, Global Acne Grading System (GAGS) score and information about the characteristics of their menstrual cycle. The GAGS score was interpreted as follows: $0=$ no acne; $1-18=$ mild acne; $19-30=$ moderate acne; $31-38$ = severe acne; $\geq 39$ = very severe acne [7]. World Health Organization AnthroPlus software was used to calculate BMI $\left(\mathrm{kg} / \mathrm{m}^{2}\right)$ and its percentile, according to age and normal range for adolescent girls [8]. Weights and heights were measured using standardised calibrated measuring devices.

Pelvic ultrasound was performed by a single examiner using either an HD11 XE or Logiq P5 ultrasound machine (Philips, Amsterdam, Netherlands; General Electric, Boston, MA, USA, respectively). Polycystic ovaries were defined as an ovarian volume $>10 \mathrm{~mL}$ in at least one ovary and no corpus luteum, dominant follicle or other cystic structure in any ovary. Ovarian volume was calculated using the simplified formula for a prolate ellipsoid. The larger ovary was used to evaluate ovarian size.

For PCOS patients, T, androstenedione (A2) and DHEA-SO4 were tested on the 3rd to 5 th day of the menstrual cycle. For patients with amenorrhea, testing was performed in any day in the morning. In order to exclude accidental testing during the periovulatory or luteal phase, if menstruation occurred during the next 21 days, repeated testing on the 3rd to 5th day of the menstrual cycle was performed. Additionally, thyroid-stimulating hormone, prolactin, luteinising hormone, follicle-stimulating hormone and oestradiol were tested on the same day of the menstrual cycle in order to exclude other causes of oligomenorrhoea. Androgen levels, including T, were tested in a certified laboratory using an electrochemiluminescence method (Cobas 6000 immunological analyser; Roche, Basel, Switzerland).

\subsection{Genetic Testing}

Peripheral venous blood samples were collected from the study participants into EDTA-containing tubes, and genomic DNA was isolated using Analytik Jena's innuPREP Blood DNA Mini Kit (Jena, Germany).

Genotyping for rs6166 (FSHR) and rs4986938 (ESR2) was performed using a multiplex PCR assay GENTERF (developed at Riga Stradins University); rs104893837 (GNRHR) was detected by Sanger sequencing using a BigDye Terminator Kit following the manufacturer's protocol and employing previously described probes (https:/ / www.academic.oup.com/ jcem/article/87/4/1607/2374953. Accessed on 1 January 2021); rs2293275 (LHCGR) and rs6165 (FSHR) were identified with PCR-RFLP methods adapted from previous publications (doi:10.1111/j.1365-2265.2012.04372.x, https:/ / www.pubmed.ncbi.nlm.nih.gov/19 387820/. Accessed on 1 January 2021); rs2349415 (FSHR) was detected by PCR-RFLP using the restriction enzyme MboI (primers are available upon request). 
Duplicates were used to assess the genotyping quality, and for each variant detected by GENTERF or PCR-RFLP, at least $10 \%$ of the samples were validated with Sanger sequencing following the manufacturer's protocol.

To exclude random error and bias in the gene-disease associations, we checked our genotype frequencies were in Hardy-Weinberg equilibrium.

\subsection{Statistical Methods}

Statistical Package for the Social Sciences software (SPSS22.0) was used for all the statistical analyses. Medians were used to describe the central tendency as the data were not normally distributed (Kolmogorov-Smirnov test $p<0.05$ ). Parametric (Student's $t$-test, ANOVA) and nonparametric (Mann-Whitney U test, Kruskal-Wallis test) tests were used for range and interval scale data, and Pearson's chi-squared test and Fisher's Exact test were used for nominal scale data. Two-tailed $p<0.05$ was considered statistically significant.

\section{Results}

The baseline clinical characteristics of the PCOS, risk and control groups are presented in Table 1. To characterise the age of participants by group, we chose a more precise characteristic of the adolescent population - gynaecological age (years since menarche)—which did not significantly differ among the groups $(p=0.441)$. Significantly more adolescents in the PCOS group and risk group showed markers of poor metabolic health. Specifically, a higher BMI, higher waist-hip ratio and higher percentage of participants with obesity were observed. BMI and waist-hip ratio were significantly higher in PCOS and risk group patients than in control group patients, accordingly $p<0.001$ and $p=0.001$. In addition, BMI above the 85th percentile was significantly more common in individuals from the PCOS group than from the control and risk groups $(p<0.001)$. Approximately one-third $(34.9 \%)$ of adolescent PCOS patients had characteristic polycystic ovary appearance on ultrasound examination, significantly $(p=0.001)$ more than in the risk and control groups (13.6\% and $7.5 \%$, respectively). Overall, acne was more pronounced in the PCOS and risk group than in the control group $(p<0.001)$, with moderate acne being the degree of severity that was significantly more common in PCOS patients $(p=0.002)$. All patients had normal prolactin and thyroid-stimulating hormone levels. Additionally, oestradiol, luteinising hormone and follicle-stimulating hormone levels were in the normal laboratory range of premenopausal women (as per inclusion criteria).

Table 1. Baseline clinical characteristics of the PCOS, risk and control groups.

\begin{tabular}{|c|c|c|c|c|}
\hline Variable & PCOS Group $(n=63)$ & Risk Group $(n=22)$ & Control Group $(n=67)$ & $p$-Value \\
\hline Gynaecological age, median years (IQR) $†$ & $3.0(2.0)$ & $4.0(2.0)$ & $4.0(1.0)$ & 0.441 \\
\hline BMI, median percentile (IQR) & $89.9(48.0)$ & $75.4(39.8)$ & $55.0(47.0)$ & $<0.001$ * \\
\hline $\begin{array}{l}\text { Individuals with BMI above the } 85 \text { th } \\
\text { percentile, } n(\%)\end{array}$ & $31(49.2)$ & $6(27.3)$ & $9(13.4)$ & $<0.001^{* *}$ \\
\hline Waist-hip ratio, median (IQR) & $0.82(0.13)$ & $0.80(0.06)$ & $0.76(0.06)$ & $0.001 *$ \\
\hline mFG score, median (IQR) & $9.0(6.0)$ & $8.0(5.0)$ & $1.0(2.0)$ & $<0.001$ * \\
\hline GAGS score, mean (SD) & $14.5(9.1)$ & $10.9(8.8)$ & $7.0(6.0)$ & $<0.001^{\wedge}$ \\
\hline No acne, $n(\%)$ & $1(1.9)$ & $2(10.0)$ & $6(15.0)$ & \\
\hline Mild acne, $n(\%)$ & $35(64.8)$ & $13(65.0)$ & $31(77.5)$ & $0.147^{* * *}$ \\
\hline Moderate acne, $n(\%)$ & $16(29.6)$ & $4(20.0)$ & $3(7.5)$ & $0.002 * * *$ \\
\hline Severe acne, $n(\%)$ & $2(3.7)$ & $1(5.0)$ & $0(0)$ & $0.091^{* * *}$ \\
\hline $\begin{array}{c}\text { Polycystic ovary morphology on } \\
\text { ultrasound, } n(\%)\end{array}$ & $22(34.9)$ & $3(13.6)$ & $5(7.5)$ & $0.001^{* * *}$ \\
\hline
\end{tabular}

+ Gynaecological age—age (in years) at the time of the study minus age at menarche; BMI—body mass index; mFG—modified FerrimanGallwey; GAGS-Global Acne Grading System; * Kruskal-Wallis test; ** Pearson's chi-squared test; *** Fisher's exact test; ^ one-way ANOVA; IQR -interquartile range for medians; statistically significant $p$-values are denoted in bold. 
The SNVs' genotype frequencies are summarized in Table 2. For all six SNVs, there were no significant differences in the genotype frequencies among the PCOS, risk and control groups.

Table 2. Genotype frequencies of the six SNVs in the PCOS, risk and control groups.

\begin{tabular}{|c|c|c|c|c|c|c|c|c|c|c|}
\hline \multirow[t]{2}{*}{ SNV/Genotype } & \multicolumn{3}{|c|}{ PCOS Group $(n=63)$} & \multicolumn{3}{|c|}{ Risk Group $(n=22)$} & \multicolumn{3}{|c|}{ Control Group $(n=67)$} & \multirow[t]{2}{*}{$p$-Value } \\
\hline & $\begin{array}{c}\mathrm{HH} \\
n(\%)\end{array}$ & $\begin{array}{l}\mathrm{Hh} \\
n(\%)\end{array}$ & $\begin{array}{c}\text { hh } \\
n(\%)\end{array}$ & $\begin{array}{c}\mathrm{HH} \\
n(\%)\end{array}$ & $\begin{array}{c}\mathrm{Hh} \\
n(\%)\end{array}$ & $\begin{array}{l}\text { hh } \\
n(\%)\end{array}$ & $\begin{array}{c}\mathrm{HH} \\
n(\%)\end{array}$ & $\begin{array}{c}\mathrm{Hh} \\
n(\%)\end{array}$ & $\begin{array}{c}\text { hh } \\
n(\%)\end{array}$ & \\
\hline FSHR rs2349415 & $\begin{array}{c}25 \\
(39.7)\end{array}$ & $\begin{array}{c}28 \\
(44.4)\end{array}$ & $\begin{array}{c}10 \\
(15.9)\end{array}$ & $7(31.8)$ & $\begin{array}{c}11 \\
(50.0)\end{array}$ & $\begin{array}{c}4 \\
(18.2)\end{array}$ & $\begin{array}{c}20 \\
(29.9)\end{array}$ & $\begin{array}{c}33 \\
(49.3)\end{array}$ & $\begin{array}{c}14 \\
(20.9)\end{array}$ & $0.81^{*}$ \\
\hline FSHR rs6166 & $\begin{array}{c}18 \\
(28.6)\end{array}$ & $\begin{array}{c}30 \\
(47.6)\end{array}$ & $\begin{array}{c}15 \\
(23.8)\end{array}$ & $7(31.8)$ & $\begin{array}{c}12 \\
(54.5)\end{array}$ & $\begin{array}{c}3 \\
(13.6)\end{array}$ & $\begin{array}{c}24 \\
(35.8)\end{array}$ & $\begin{array}{c}29 \\
(43.3)\end{array}$ & $\begin{array}{c}14 \\
(20.9)\end{array}$ & $0.77^{*}$ \\
\hline FSHR rs6165 & $\begin{array}{c}15 \\
(23.8)\end{array}$ & $\begin{array}{c}36 \\
(57.1)\end{array}$ & $\begin{array}{c}12 \\
(19.1)\end{array}$ & $5(22.7)$ & $\begin{array}{c}13 \\
(59.1)\end{array}$ & $\begin{array}{c}4 \\
(18.2)\end{array}$ & $\begin{array}{c}21 \\
(31.3)\end{array}$ & $\begin{array}{c}31 \\
(46.3)\end{array}$ & $\begin{array}{c}15 \\
(22.4)\end{array}$ & $0.73^{*}$ \\
\hline ESR2 rs4986938 & $\begin{array}{c}14 \\
(22.2)\end{array}$ & $\begin{array}{c}38 \\
(60.3)\end{array}$ & $\begin{array}{c}11 \\
(17.5)\end{array}$ & $5(22.7)$ & $\begin{array}{c}13 \\
(59.1)\end{array}$ & $\begin{array}{c}4 \\
(18.2)\end{array}$ & $\begin{array}{c}10 \\
(14.9)\end{array}$ & $\begin{array}{c}49 \\
(73.1)\end{array}$ & $\begin{array}{c}8 \\
(11.9)\end{array}$ & $0.58^{*}$ \\
\hline $\begin{array}{c}\text { GNRHR } \\
\text { rs104893837 }\end{array}$ & $\begin{array}{c}59 \\
(93.7)\end{array}$ & $\begin{array}{c}4 \\
(6.3)\end{array}$ & $\begin{array}{c}0 \\
(0)\end{array}$ & $21(95.5)$ & $\begin{array}{c}1 \\
(4.5)\end{array}$ & $\begin{array}{c}0 \\
(0)\end{array}$ & $\begin{array}{c}64 \\
(95.5)\end{array}$ & $\begin{array}{c}3 \\
(4.5)\end{array}$ & $\begin{array}{c}0 \\
(0)\end{array}$ & $0.89^{* *}$ \\
\hline LHCGR rs2293275 & $\begin{array}{c}26 \\
(41.3)\end{array}$ & $\begin{array}{c}26 \\
(41.3)\end{array}$ & $\begin{array}{c}11 \\
(17.4)\end{array}$ & $6(27.3)$ & $\begin{array}{c}11 \\
(50.0)\end{array}$ & $\begin{array}{c}5 \\
(22.7)\end{array}$ & $\begin{array}{c}22 \\
(32.8)\end{array}$ & $\begin{array}{c}31 \\
(46.3)\end{array}$ & $\begin{array}{c}14 \\
(20.9)\end{array}$ & $0.77^{*}$ \\
\hline
\end{tabular}

* Pearson's chi-squared test; ${ }^{* *}$ Fisher's exact test; HH-homozygous carriers of major alleles; Hh-heterozygous allele carriers; hhhomozygous carriers of minor alleles.

The associations of the PCOS patients' genotype and phenotypic parameters are shown in Table 3. ESR2 rs4986938 homozygous minor allele carriers had a significantly higher level of $\mathrm{T}(0.68 \mathrm{ng} / \mathrm{mL})$ than heterozygous individuals $(0.38 \mathrm{ng} / \mathrm{mL})$ and homozygous major allele carriers $(0.38 \mathrm{ng} / \mathrm{mL})(p=0.04)$. There was a tendency towards a lower $\mathrm{mFG}$ score in ESR2 rs4986938 heterozygous individuals; however, this result did not quite reach statistical significance $(p=0.056)$.

The phenotype associations with homozygous major allele carriers $(\mathrm{HH})$ and minor allele carriers (Hh and $\mathrm{hh}$ ) in the PCOS group are shown in Table 4.

We found that the level of T was significantly higher in LHCGR rs2293275 minor allele carriers $(0.61 \mathrm{ng} / \mathrm{mL})$ than homozygous major allele carriers $(0.35 \mathrm{ng} / \mathrm{mL})(p=0.044)$. LHCGR rs2293275 minor allele carriers also demonstrated a tendency towards a higher waist-hip ratio compared with homozygous major allele carriers; however, this result did not reach statistical significance $(p=0.08)$. Similarly, although statistical significance was not achieved ( $p=0.084)$, FSHR rs6166 minor allele carriers tended towards more severe acne (higher GAGS score) compared with homozygous major allele carriers. 
Table 3. Genotype-phenotype associations in PCOS patients.

\begin{tabular}{|c|c|c|c|c|c|c|c|c|c|c|c|c|c|c|c|c|c|c|c|c|c|c|c|c|}
\hline \multirow{2}{*}{ SNV } & \multicolumn{4}{|c|}{$\begin{array}{l}\text { Modified Ferriman-Gallwey } \\
\text { Score, Median (IQR) }\end{array}$} & \multicolumn{4}{|c|}{ BMI Percentile, Median (IQR) } & \multicolumn{4}{|c|}{$\begin{array}{c}\text { Waist-Hip Ratio, Median } \\
\text { (IQR) }\end{array}$} & \multicolumn{4}{|c|}{$\begin{array}{c}\text { Total Testosterone Level, } \\
\text { Median (IQR) }\end{array}$} & \multicolumn{4}{|c|}{ GAGS Score, Mean (SD) } & \multicolumn{4}{|c|}{$\begin{array}{l}\text { PCO Morphology on } \\
\text { Ultrasound, } n(\%)\end{array}$} \\
\hline & HH & Hh & hh & $p^{*}$ & HH & $\mathrm{Hh}$ & hh & $p^{*}$ & HH & $\mathrm{Hh}$ & hh & $p^{*}$ & HH & Hh & hh & $p^{*}$ & HH & Hh & hh & $p^{\wedge}$ & HH & Hh & hh & $p$ \\
\hline $\begin{array}{c}\text { FSHR } \\
\text { rs2349415 }\end{array}$ & $\begin{array}{l}10.0 \\
(6.0)\end{array}$ & $\begin{array}{c}9.0 \\
(6.0)\end{array}$ & $\begin{array}{l}12.5 \\
(9.8)\end{array}$ & 0.44 & $\begin{array}{c}82.2 \\
(64.1)\end{array}$ & $\begin{array}{c}93.1 \\
(45.0)\end{array}$ & $\begin{array}{c}91.4 \\
(26.1)\end{array}$ & 0.88 & $\begin{array}{c}0.84 \\
(0.19)\end{array}$ & $\begin{array}{c}0.84 \\
(0.11)\end{array}$ & $\begin{array}{c}0.77 \\
(0.17)\end{array}$ & 0 & $\begin{array}{c}0.42 \\
(0.37)\end{array}$ & $\begin{array}{c}0.48 \\
(0.52)\end{array}$ & $\begin{array}{c}0.40 \\
(0.37)\end{array}$ & 0 & $\begin{array}{l}15.0 \\
(10.7)\end{array}$ & $\begin{array}{l}13.8 \\
(7.6)\end{array}$ & $\begin{array}{l}13.5 \\
(8.1)\end{array}$ & 9 & $\begin{array}{c}9 \\
(40.9)\end{array}$ & $\begin{array}{c}10 \\
(45.5)\end{array}$ & $\begin{array}{c}3 \\
(13.6)\end{array}$ & $\underset{* *}{0.849}$ \\
\hline $\begin{array}{l}\text { FSHR } \\
\text { rs6166 }\end{array}$ & $\begin{array}{c}9.0 \\
(7.5)\end{array}$ & $\begin{array}{c}9.5 \\
(6.0)\end{array}$ & $\begin{array}{c}14.0 \\
(10.5)\end{array}$ & 0.19 & $\begin{array}{l}91.1) \\
95.6 \\
(60.6)\end{array}$ & $\begin{array}{c}76.7 \\
(46.6)\end{array}$ & $\begin{array}{l}81.8 \\
81.8 \\
(60.4)\end{array}$ & 0.93 & $\begin{array}{c}0.83 \\
(0.17)\end{array}$ & $\begin{array}{c}0.82 \\
(0.13)\end{array}$ & $\begin{array}{c}0.84 \\
(0.10)\end{array}$ & 0.89 & $\begin{array}{c}0.53 \\
(0.32)\end{array}$ & $\begin{array}{c}0.38 \\
(0.44)\end{array}$ & $\begin{array}{c}0.44 \\
(0.42)\end{array}$ & 0.80 & $\begin{array}{l}10.4 \\
(7.8)\end{array}$ & $\begin{array}{l}16.5 \\
(9.4)\end{array}$ & $\begin{array}{l}14.0 \\
(8.4)\end{array}$ & 0.12 & $\begin{array}{c}5 \\
(22.7)\end{array}$ & $\begin{array}{c}10 \\
(45.5)\end{array}$ & $\begin{array}{c}7 \\
(31.8)\end{array}$ & $\underset{* *}{0.371}$ \\
\hline $\begin{array}{l}\text { FSHR } \\
\text { rs6165 }\end{array}$ & $\begin{array}{c}9.0 \\
(7.0)\end{array}$ & $\begin{array}{c}9.0 \\
(6.0)\end{array}$ & $\begin{array}{l}15.0 \\
(8.0)\end{array}$ & 0.12 & $\begin{array}{l}95.6 \\
(63.9)\end{array}$ & $\begin{array}{c}76.7 \\
(48.0)\end{array}$ & $\begin{array}{l}94.0 \\
(41.4)\end{array}$ & 0.71 & $\begin{array}{c}0.84 \\
(0.16)\end{array}$ & $\begin{array}{c}0.82 \\
(0.14)\end{array}$ & $\begin{array}{c}0.84 \\
(0.13)\end{array}$ & 0.94 & $\begin{array}{c}0.45 \\
(0.49)\end{array}$ & $\begin{array}{c}0.40 \\
(0.41)\end{array}$ & $\begin{array}{c}0.57 \\
(0.43)\end{array}$ & 0 & $\begin{array}{l}12.4 \\
(8.8)\end{array}$ & $\begin{array}{l}15.1 \\
(9.3)\end{array}$ & $\begin{array}{l}13.4 \\
(9.2)\end{array}$ & 0.70 & $\begin{array}{c}4 \\
(18.2)\end{array}$ & $\begin{array}{c}12 \\
(54.5)\end{array}$ & $\begin{array}{c}6 \\
(27.3)\end{array}$ & $\underset{* * *}{0.492}$ \\
\hline $\begin{array}{c}\text { ESR2 } \\
\text { rs4986938 }\end{array}$ & $\begin{array}{l}10.0 \\
(6.0)\end{array}$ & $\begin{array}{c}8.0 \\
(7.3)\end{array}$ & $\begin{array}{l}11.0 \\
(6.5)\end{array}$ & 0.056 & $\begin{array}{c}90.4 \\
(49.9)\end{array}$ & $\begin{array}{l}89.0 \\
(53.9)\end{array}$ & $\begin{array}{l}96.6 \\
(30.8)\end{array}$ & 0.36 & $\begin{array}{c}0.81 \\
(0.09)\end{array}$ & $\begin{array}{c}0.81 \\
(0.14)\end{array}$ & $\begin{array}{c}0.85 \\
(0.05)\end{array}$ & 0.63 & $\begin{array}{c}0.38 \\
(0.26)\end{array}$ & $\begin{array}{c}0.38 \\
(0.38)\end{array}$ & $\begin{array}{c}0.68 \\
(0.20)\end{array}$ & 0.04 & $\begin{array}{l}14.0 \\
(10.9)\end{array}$ & $\begin{array}{c}13.3 \\
(15.3)\end{array}$ & $\begin{array}{c}13.4 \\
(16.5)\end{array}$ & 0.71 & $\begin{array}{c}6 \\
(27.3)\end{array}$ & $\begin{array}{c}13 \\
(59.1)\end{array}$ & $\begin{array}{c}3 \\
(13.6)\end{array}$ & $\underset{* * *}{0.791}$ \\
\hline $\begin{array}{l}\text { GNRHR } \\
\text { rs104893837 }\end{array}$ & $\begin{array}{c}9.5 \\
(6.0)\end{array}$ & 10.0 & $\begin{array}{c}0 \\
(0)\end{array}$ & 0.99 & $\begin{array}{c}90.4 \\
(48.0)\end{array}$ & 74.9 & $\begin{array}{c}0 \\
(0)\end{array}$ & 0.75 & $\begin{array}{c}0.83 \\
(0.13)\end{array}$ & 0.88 & $\begin{array}{c}0 \\
(0)\end{array}$ & 0.97 & $\begin{array}{c}0.44 \\
(0.40)\end{array}$ & 0.33 & $\begin{array}{c}0 \\
(0)\end{array}$ & 0.47 & $\begin{array}{l}14.4 \\
(8.9)\end{array}$ & $\begin{array}{c}12.5 \\
(16.3)\end{array}$ & $\begin{array}{c}0 \\
(0)\end{array}$ & 0.31 & $\begin{array}{c}21 \\
(95.5)\end{array}$ & $\begin{array}{c}1 \\
(4.5)\end{array}$ & $\begin{array}{c}0 \\
(0)\end{array}$ & $\underset{* * *}{1.000}$ \\
\hline $\begin{array}{c}\text { LHCGR } \\
\text { rs2293275 }\end{array}$ & $\begin{array}{c}9.0 \\
(6.0)\end{array}$ & $\begin{array}{l}10.5 \\
(5.8)\end{array}$ & $\begin{array}{l}14.0 \\
(12.0)\end{array}$ & 0.92 & $\begin{array}{l}71.6 \\
(56.8)\end{array}$ & $\begin{array}{c}89.0 \\
(49.4)\end{array}$ & $\begin{array}{c}98.9 \\
(15.9)\end{array}$ & 0.27 & $\begin{array}{c}0.81 \\
(0.11)\end{array}$ & $\begin{array}{c}0.84 \\
(0.17)\end{array}$ & $\begin{array}{c}0.88 \\
(0.09)\end{array}$ & 0.19 & $\begin{array}{c}0.35 \\
(0.37)\end{array}$ & $\begin{array}{c}0.59 \\
(0.36)\end{array}$ & $\begin{array}{c}0.67 \\
(0.66)\end{array}$ & 0.13 & $\begin{array}{l}14.3 \\
(9.0)\end{array}$ & $\begin{array}{l}15.6 \\
(9.6)\end{array}$ & $\begin{array}{l}10.6 \\
(7.6)\end{array}$ & 0.60 & $\begin{array}{c}9 \\
(40.9)\end{array}$ & $\begin{array}{c}9 \\
(40.9)\end{array}$ & $\begin{array}{c}4 \\
(18.2)\end{array}$ & $\underset{* * *}{0.814}$ \\
\hline
\end{tabular}

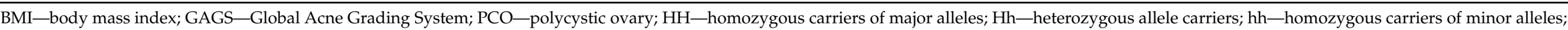

${ }^{*}$ Kruskal-Wallis test; ** Pearson's chi-squared test; ${ }^{* * *}$ Fisher's exact test ${ }^{\wedge}$ one-way ANOVA; IQR-interquartile range for medians; statistically significant $p$-value is denoted in bold.

Table 4. Phenotype associations with homozygous major $(\mathrm{HH})$ and minor $(\mathrm{Hh} / \mathrm{hh})$ allele carriers in the PCOS group.

\begin{tabular}{|c|c|c|c|c|c|c|c|c|c|c|c|c|c|c|c|c|c|c|}
\hline \multirow[b]{2}{*}{ SNV } & \multicolumn{3}{|c|}{$\begin{array}{c}\text { Modified Ferriman-Gallwey Score, } \\
\text { Median (IQR) }\end{array}$} & \multicolumn{3}{|c|}{ BMI Percentile, Median (IQR) } & \multicolumn{3}{|c|}{ Waist-Hip Ratio, Median (IQR) } & \multicolumn{3}{|c|}{$\begin{array}{c}\text { Total Testosterone Level, Median } \\
\text { (IQR) }\end{array}$} & \multicolumn{3}{|c|}{ GAGS Score, Mean (SD) } & \multicolumn{3}{|c|}{ PCO Morphology on Ultrasound, $n(\%$} \\
\hline & $\begin{array}{l}\text { Major } \\
\text { Allele Ho- } \\
\text { mozygotes } \\
\text { (HH) }\end{array}$ & $\begin{array}{l}\text { Minor } \\
\text { Allele } \\
\text { Carriers } \\
\text { (Hh, hh) }\end{array}$ & $p^{*}$ & $\begin{array}{c}\text { Major } \\
\text { Allele Ho- } \\
\text { mozygotes } \\
(\mathrm{HH})\end{array}$ & $\begin{array}{l}\text { Minor } \\
\text { Allele } \\
\text { Carriers } \\
\text { (Hh, hh) }\end{array}$ & $p^{*}$ & $\begin{array}{c}\text { Major } \\
\text { Allele Ho- } \\
\text { mozygotes } \\
\text { (HH) }\end{array}$ & $\begin{array}{l}\text { Minor } \\
\text { Allele } \\
\text { Carriers } \\
\text { (Hh, hh) }\end{array}$ & $p^{*}$ & $\begin{array}{l}\text { Major } \\
\text { Allele Ho- } \\
\text { mozygotes } \\
\text { (HH) }\end{array}$ & $\begin{array}{l}\text { Minor } \\
\text { Allele } \\
\text { Carriers } \\
\text { (Hh, hh) }\end{array}$ & $p^{*}$ & $\begin{array}{l}\text { Major } \\
\text { Allele Ho- } \\
\text { mozygotes } \\
\text { (HH) }\end{array}$ & $\begin{array}{l}\text { Minor } \\
\text { Allele } \\
\text { Carriers } \\
\text { (Hh, hh) }\end{array}$ & $p^{\wedge}$ & $\begin{array}{c}\text { Major } \\
\text { Allele Ho- } \\
\text { mozygotes } \\
\text { (HH) }\end{array}$ & $\begin{array}{l}\text { Minor } \\
\text { Allele } \\
\text { Carriers } \\
\text { (Hh, hh) }\end{array}$ & $p$ \\
\hline $\begin{array}{l}\text { FSHR } \\
\text { rs2349415 }\end{array}$ & $\begin{array}{l}10.0 \\
(6.0)\end{array}$ & $\begin{array}{c}9.5 \\
(7.8)\end{array}$ & 0.84 & $\begin{array}{l}82.2 \\
(64.1)\end{array}$ & $\begin{array}{c}92.3 \\
(41.2)\end{array}$ & 0.73 & $\begin{array}{l}0.84 \\
(0.19)\end{array}$ & $\begin{array}{c}0.83 \\
(0.12)\end{array}$ & 0.36 & $\begin{array}{c}0.42 \\
(0.37)\end{array}$ & $\begin{array}{l}0.46 \\
(0.47)\end{array}$ & 0.90 & $\begin{array}{l}14.9 \\
(10.7)\end{array}$ & $\begin{array}{l}14.0 \\
(7.6)\end{array}$ & 0.902 & $\begin{array}{c}9 \\
(40.9)\end{array}$ & $\begin{array}{c}13 \\
(59.1)\end{array}$ & $0.879^{* *}$ \\
\hline $\begin{array}{l}\text { FSHR } \\
\text { rs6166 }\end{array}$ & $\begin{array}{c}9.0 \\
(7.5)\end{array}$ & $\begin{array}{l}10.0 \\
(7.0)\end{array}$ & 0.60 & $\begin{array}{l}95.6 \\
(60.6)\end{array}$ & $\begin{array}{l}76.7 \\
(48.1)\end{array}$ & 0.73 & $\begin{array}{c}0.83 \\
(0.17)\end{array}$ & $\begin{array}{c}0.84 \\
(0.13)\end{array}$ & 0.90 & $\begin{array}{c}0.53 \\
(0.32)\end{array}$ & $\begin{array}{c}0.38 \\
(0.42)\end{array}$ & 0.50 & $\begin{array}{l}10.4 \\
(7.8)\end{array}$ & $\begin{array}{l}16.3 \\
(9.1)\end{array}$ & 0.084 & $\begin{array}{c}5 \\
(22.7)\end{array}$ & $\begin{array}{c}17 \\
(77.3)\end{array}$ & $0.389 * *$ \\
\hline $\begin{array}{l}\text { FSHR } \\
\text { rs6165 }\end{array}$ & $\begin{array}{c}9.0 \\
(7.0) \\
\end{array}$ & $\begin{array}{l}10.0 \\
(6.0)\end{array}$ & 0.64 & $\begin{array}{l}95.6 \\
(63.9) \\
\end{array}$ & $\begin{array}{c}83.7 \\
(48.1) \\
\end{array}$ & 0.79 & $\begin{array}{c}0.84 \\
(0.16) \\
\end{array}$ & $\begin{array}{c}0.83 \\
(0.14) \\
\end{array}$ & 0.98 & $\begin{array}{c}0.45 \\
(0.49) \\
\end{array}$ & $\begin{array}{c}0.43 \\
(0.39) \\
\end{array}$ & 0.74 & $\begin{array}{l}12.4 \\
(8.6)\end{array}$ & $\begin{array}{l}15.1 \\
(9.2) \\
\end{array}$ & 0.434 & $\begin{array}{c}4 \\
(18.2) \\
\end{array}$ & $\begin{array}{c}18 \\
(81.8) \\
\end{array}$ & $0.747^{* * *}$ \\
\hline $\begin{array}{c}\text { ESR2 } \\
\text { rs4986938 }\end{array}$ & $\begin{array}{l}10.0 \\
(6.0)\end{array}$ & $\begin{array}{c}8.0 \\
(7.0)\end{array}$ & 0.07 & $\begin{array}{c}90.4 \\
(49.9)\end{array}$ & $\begin{array}{c}89.4 \\
(47.1)\end{array}$ & 0.92 & $\begin{array}{c}0.81 \\
(0.09)\end{array}$ & $\begin{array}{c}0.83 \\
(0.12)\end{array}$ & 0.45 & $\begin{array}{c}0.38 \\
(0.26)\end{array}$ & $\begin{array}{c}0.47 \\
(0.42)\end{array}$ & 0.40 & $\begin{array}{l}14.0 \\
(10.9)\end{array}$ & $\begin{array}{l}13.3 \\
(8.7)\end{array}$ & 0.476 & $\begin{array}{c}6 \\
(27.3)\end{array}$ & $\begin{array}{c}16 \\
(72.7)\end{array}$ & $0.566^{* * *}$ \\
\hline $\begin{array}{l}\text { GNRHR } \\
\text { rs104893837 }\end{array}$ & $\begin{array}{l}10.0 \\
(6.0)\end{array}$ & 10.0 & 0.99 & $\begin{array}{c}90.4 \\
(48.0)\end{array}$ & 74.9 & 0.75 & $\begin{array}{c}0.84 \\
(0.12) \\
\end{array}$ & 0.88 & 0.97 & $\begin{array}{c}0.45 \\
(0.40) \\
\end{array}$ & 0.33 & 0.47 & $\begin{array}{l}14.5 \\
(9.0)\end{array}$ & $\begin{array}{c}12.5 \\
(16.3)\end{array}$ & 0.312 & $\begin{array}{c}21 \\
(95.5)\end{array}$ & $\begin{array}{c}1 \\
(4.5) \\
\end{array}$ & $1.000^{* * *}$ \\
\hline $\begin{array}{c}\text { LHCGR } \\
\text { rs2293275 }\end{array}$ & $\begin{array}{c}9.0 \\
(6.0)\end{array}$ & $\begin{array}{l}11.5 \\
(6.3)\end{array}$ & 0.71 & $\begin{array}{c}71.6 \\
(56.8)\end{array}$ & $\begin{array}{c}94.4 \\
(44.5)\end{array}$ & 0.37 & $\begin{array}{c}0.81 \\
(0.11)\end{array}$ & $\begin{array}{c}0.86 \\
(0.11)\end{array}$ & 0.08 & $\begin{array}{c}0.35 \\
(0.37)\end{array}$ & $\begin{array}{c}0.61 \\
(0.42)\end{array}$ & 0.044 & $\begin{array}{l}14.3 \\
(9.0)\end{array}$ & $\begin{array}{l}14.5 \\
(9.4)\end{array}$ & 0.902 & $\begin{array}{c}9 \\
(40.9)\end{array}$ & $\begin{array}{c}13 \\
(59.1)\end{array}$ & $0.792 * *$ \\
\hline
\end{tabular}

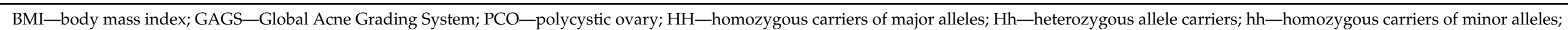

${ }^{*}$ Kruskal-Wallis test; ${ }^{* *}$ Pearson's chi-squared test; ${ }^{* * *}$ Fisher's exact test; ${ }^{\wedge}$ Student's $t$-test; IQR-interquartile range for medians; statistically significant $p$-value is denoted in bold. 


\section{Discussion}

For all six SNVs investigated in the present study-GNRHR (rs104893837), ESR2 (rs4986938), LHCGR (rs2293275) and FSHR (rs6166, rs6165, rs2349415)- there were no significant differences in the genotype frequencies among the PCOS, risk and control groups. Within the PCOS group, patients who were ESR2 rs4986938 homozygous minor allele carriers had a significantly higher level of $\mathrm{T}$ than heterozygous individuals and homozygous major allele carriers $(p=0.04)$. In addition, the level of T was significantly higher in PCOS patients who were LHCGR rs2293275 minor allele carriers than in those who were homozygous major allele carriers $(p=0.044)$. The genotype-phenotype associations of GNRHR (rs104893837) and FSHR (rs6166, rs6165, rs2349415) in PCOS patients did not reach statistical significance.

The ESR2 gene is located at chromosome 14q23.2q23.3. It encodes oestrogen receptor 2-a steroid receptor situated in the cell nucleus. ESR2 rs4986938 is positioned in the non-coding sequence of the gene. Due to ESR2's role in ovarian function, it has been studied in PCOS patients and differences in its ovarian expression level have been reported between patients and controls $[9,10]$. Several research groups have investigated this SNV's association with PCOS and its symptoms in different ethnic populations. For instance, Douma and colleagues observed a lower level of hyperandrogenism in Tunisian PCOS patients who were minor allele carriers; however, this association was lost following Bonferroni correction [11]. Indeed, the majority of studies have not found an association between minor allele carrier status and PCOS and its symptoms [11-15]. Nevertheless, Kim et al. found that minor allele carriers had a lower risk of developing PCOS than homozygous major allele carriers in a sample of Korean women [16]. In contrast, Liaqat and co-workers' study of Pakistani women reported a higher percentage of minor allele carriers among PCOS patients than controls [17]. The mechanism of how this SNV leads to a higher level of testosterone still needs to be elucidated. One suggestion derived from similar results investigating SNV in ESR1 gene is that the lower expression of ESR could increase the conversion of androgen precursors to testosterone in PCOS women [18].

We found that PCOS patients who were LHCGR rs2293275 minor allele carriers had a significantly higher $\mathrm{T}$ level than major allele homozygotes. This finding was not affected by the BMI of the participants; however, the association was lost when adjustment for waist-hip ratio was performed. The LHCGR gene is located at chromosome $2 \mathrm{p} 16.3$. It encodes a receptor for both luteinising hormone and choriogonadotropin hormone. The gene is expressed in different cell types in the ovary, including theca cells and differentiated granulosa cells, and LHCGR is thought to transduce luteinising hormone-mediated signals that play a crucial role in the ovulation process [19]. Consequently, if an increased reaction to the circulating level of luteinising hormone occurs, normal follicular development and ovulation are impaired. At the same time, the expression of aromatase increases, facilitating androgen production [20]. The published data regarding rs2293275's role in the development of PCOS are contradictory. Valkenburg and colleagues did not find an association between this SNV and PCOS development in a European population [21]. However, a meta-analysis conducted by Zou et al. reported a 4.1 risk increase of developing PCOS for carriers of the AA (minor allele) genotype in a Caucasian population [22]. Findings also vary regarding the SNV's role in the phenotype of PCOS patients. Thathapudi et al.'s study of South Indian women detected an association of the GG (major allele) genotype with BMI, waist-hip ratio, insulin resistance, luteinising hormone level and luteinising hormone/follicle-stimulating hormone ratio in PCOS patients compared with controls. The AA genotype, significant in our study, was linked to a higher basal follicle-stimulating hormone level than non-A allele carriers. The T level was not reported in this particular study [23]. El-Shal et al.'s study of Egyptian PCOS women found an association between LHCGR rs2293275 and several anthropometric and biochemical characteristics, including elevated values of the free androgen index and hirsutism score [24]. A study of European PCOS women (The Netherlands) reported a lower basal follicle-stimulating hormone level in patients who had neither the LHCGR rs2293275 SNV nor 18insLQ SNV; however, 
no other clinical associations were detected (14). Another study of a European population (Sardinia) did not find any associations of the SNV with the clinical presentation of PCOS [25].

There were no significant differences in the genotype and allele carrier frequencies of FSHR (rs6166, rs6165, rs2349415) among our PCOS, risk and control groups. FSHR is a G protein-coupled receptor in the cell membrane of ovarian granulosa cells. Several SNVs in this gene have been described as possible precursors for the development of PCOS. The SNVs rs6166 and rs6165, which result in a change of amino acid in FSHR [26], are amongst the most extensively studied variants. Genetic association studies conducted in European populations have reported a link between rs6166 and rs6165 [27] or rs6165 alone [28] and PCOS. Qiu et al. detected a similar association in their candidate gene systematic review of women of European ancestry. They concluded, rs6166 AA (major allele) carriers were less likely to develop PCOS (OR 0.64, 95\% CI: 0.42-0.98). However, they did not find an association between rs6165 and the development of PCOS [29]. GWAS performed to date have failed to show statistically significant signals from these SNVs that are related to PCOS [4]. Although both SNVs have been found to be linked to the level of folliclestimulating hormone in PCOS patients in populations with different ethnic backgrounds, the published data on their role in other clinical and biochemical features of PCOS are contradictory, with the majority showing no association $[20,27,29,30]$. FSHR rs2349415 is located in an intron of the FSHR gene. A GWAS performed by Shi et al. found that this SNV was related to PCOS in a Han Chinese population [31]. This link has been confirmed by a meta-analysis of a European population [32] and by a family association study of a Han Chinese population [33].

We did not find a link between GNRHR rs104893837 and the development of PCOS or its associated symptoms in our study population. From sequencing of the whole coding part of the GNRHR gene, rs104893837 was found to be the most frequent variant in the Latvian population (unpublished results). In humans, GNRH executes its function through the transmembrane G protein-coupled receptor (GNRHR) mainly in the hypophysis but also in ovary and breast tissue. GNRH's main role is in normal menstrual cycle regulation via stimulation of the luteinising hormone and follicle-stimulating hormone secretion [34] and participation in the formation and atresia of the corpus luteum. One of the main pathophysiological mechanisms underlying the development of PCOS is an increase of the pulse and frequency of GNRH secretion [35]. Several gene association studies have identified numerous SNV associations with the syndrome or with its symptoms [35-39]. Despite the fact that the SNVs we analysed did not show statistically different frequency between PCOS, risk group and control group patients, some alleles were associated with higher T levels in PCOS patients. That could indicate the role of epigenetic factors (e.g., DNA methylation, histone modification) in these alleles in the development of the syndrome and its phenotype. Further research would be necessary to address these aspects.

The differences between the results presented here and previously published ones may be due to different ethnic backgrounds of the studied populations that could influence genetic differences. Furthermore, incongruencies in the diagnostic criteria applied in different studies can influence results. In our study, the PCOS patients were selected using the most recent diagnostic criteria specifically designed for adolescents. Diagnostic criteria have changed significantly over the years, starting with the $1990 \mathrm{NIH}$ criteria [40], then the 2003 Rotterdam criteria [41] and currently the ESHRE 2018 criteria based on rigorous clinical and risk analysis [1]. Therefore, it is very important to use the latest diagnostic criteria with consistency to build a reliable research knowledge base. Re-evaluation of published results to identify at risk patients is very important as these patients may develop symptoms at a later stage of life and ultimately represent a specific subtype of PCOS.

This research has some important strengths and limitations. To the best of our knowledge, this is one of the first genetic studies that have applied the latest ESHRE 2018 criteria. One important change in these diagnostic criteria is that polycystic ovarian morphology is no longer considered as diagnostic criteria in adolescent PCOS patients. It is because 
relatively large ovaries with numerous follicles is a normal finding in this age group [1]. Additionally, ovary appearance in the ultrasound does not directly correlate with PCOS symptoms, that is also demonstrated by the results in our study where $65.1 \%$ of PCOS patients did not have a polycystic ovary morphology in ultrasound, but they still exhibited other PCOS symptoms. The adolescents were recruited from the Children's Clinical University Hospital in Riga, which is the central children's hospital in Latvia where most of the country's paediatric gynaecology services are carried out free of charge and without referral. Therefore, demographic and socioeconomic backgrounds did not influence the selection of participants, thereby providing a study group representative of the whole population. Moreover, all the participants forming the three different groups underwent an identical thorough examination, allowing them to be directly compared with one another. A further major strength of our study is the inclusion of a risk group comprising of patients who have a high chance of developing PCOS at a later stage of life, thus avoiding a skew towards the most severe phenotypes presenting in adolescence. Adolescents in the risk group require close monitoring, and genetic testing is essential to understand the development of particular phenotypes and provide a prognosis for the future course of the disease. A limitation of the study is its small sample size. We intend to test a larger sample and include other SNVs, as well as conduct a follow-up for the current sample (especially the risk patients).

\section{Conclusions}

No statistically significant differences were found in the distributions of genotypes for GNRHR (rs104893837), ESR2 (rs4986938), LHCGR (rs2293275) and FSHR (rs6166, rs6165, rs2349415) among PCOS patients, risk patients and healthy controls. Within the PCOS group, patients who were ESR2 rs4986938 homozygous minor allele carriers had a significantly higher level of $\mathrm{T}$ than heterozygous individuals and homozygous major allele carriers. In addition, the level of T was significantly higher in PCOS patients who were LHCGR rs2293275 minor allele carriers than in those who were homozygous major allele carriers. The genotype-phenotype associations of GNRHR (rs104893837) and FSHR (rs6166, rs6165, rs2349415) in PCOS patients did not reach statistical significance.

Author Contributions: Conceptualization, L.L., G.L. and L.G.; methodology, L.L., G.L., L.G., Z.D. and A.K.-U.; software, L.L. and L.B.; genotyping, L.B., A.R., J.I., Z.D., I.K. and L.G.; formal analysis, L.L. and A.K.-U.; data curation, L.L., L.B. and A.K.-U.; writing-original draft preparation, L.L. and L.B.; writing-review and editing, G.L., L.G., A.K.-U., I.K. and I.D.-K.; visualization, L.L. and L.B.; funding acquisition, L.G. All authors have read and agreed to the published version of the manuscript.

Funding: The study was financially supported by a Riga Stradins University internal research grant.

Institutional Review Board Statement: The study was conducted according to the guidelines of the Declaration of Helsinki and approved by the Central Medical Ethics Committee of Latvia (protocol no. 1/16-04-12 and 3/21-02-17).

Informed Consent Statement: Informed consent was obtained from all subjects involved in the study.

Data Availability Statement: Data available on request due to restrictions.

Conflicts of Interest: The authors declare no conflict of interest.

\section{References}

1. Teede, H.J.; Misso, M.L.; Costello, M.F.; Dokras, A.; Laven, J.; Moran, L.; Piltonen, T.; Norman, R.; Andersen, M.; Azziz, R.; et al. Recommendations from the international evidence-based guideline for the assessment and management of polycystic ovary syndrome. Fertil. Steril. 2018, 110, 364-379. [CrossRef] [PubMed]

2. Legro, R.; Driscoll, D.; Strauss, J.F.; Fox, J.; Dunaif, A. Evidence for a genetic basis for hyperandrogenemia in polycystic ovary syndrome. Proc. Natl. Acad. Sci. USA 1998, 95, 14956-14960. [CrossRef] [PubMed]

3. Vink, J.M.; Sadrzadeh, S.; Lambalk, C.B.; Boomsma, D.I. Heritability of Polycystic Ovary Syndrome in a Dutch Twin-Family Study. J. Clin. Endocrinol. Metab. 2006, 91, 2100-2104. [CrossRef] [PubMed] 
4. Hiam, D.; Moreno-Asso, A.; Teede, H.J.; Laven, J.S.; Stepto, N.K.; Moran, L.J.; Gibson-Helm, M. The Genetics of Polycystic Ovary Syndrome: An Overview of Candidate Gene Systematic Reviews and Genome-Wide Association Studies. J. Clin. Med. 2019, 8, 1606. [CrossRef] [PubMed]

5. De Melo, A.S.; Dias, S.V.; Cavalli, R.D.C.; Cardoso, V.C.; Bettiol, H.; Barbieri, M.A.; Ferriani, R.A.; Vieira, C.S.; Melo, A.S.D. Pathogenesis of polycystic ovary syndrome: Multifactorial assessment from the foetal stage to menopause. Reproduction 2015, 150, R11-R24. [CrossRef]

6. Unsal, T.; Konac, E.; Yesilkaya, E.; Yilmaz, A.; Bideci, A.; Onen, H.I.; Cinaz, P.; Menevse, A. Genetic polymorphisms of FSHR, CYP17, CYP1A1, CAPN10, INSR, SERPINE1 genes in adolescent girls with polycystic ovary syndrome. J. Assist. Reprod. Genet. 2009, 26, 205-216. [CrossRef]

7. Doshi, A.; Zaheer, A.; Stiller, M.J. A comparison of current acne grading systems and proposal of a novel system. Int. J. Dermatol. 1997, 36, 416-418. [CrossRef]

8. World Health Organization. AnthroPlus for Personal Computers. Manual: Software for Assessing Growth of the World's Children and Adolescents; WHO: Geneva, Switherland, 2009.

9. Jakimiuk, A.J.; Weitsman, S.R.; Yen, H.-W.; Bogusiewicz, M.; Magoffin, D.A. Estrogen Receptor $\alpha$ and $\beta$ Expression in Theca and Granulosa Cells from Women with Polycystic Ovary Syndrome. J. Clin. Endocrinol. Metab. 2002, 87, 5532-5538. [CrossRef]

10. Artimani, T.; Saidijam, M.; Aflatoonian, R.; Amiri, I.; Ashrafi, M.; Shabab, N.; Mohammadpour, N.; Mehdizadeh, M. Estrogen and progesterone receptor subtype expression in granulosa cells from women with polycystic ovary syndrome. Gynecol. Endocrinol. 2015, 31, 379-383. [CrossRef]

11. Douma, Z.; Dallel, M.; Bahia, W.; Ben Salem, A.; Ben Ali, F.H.; Almawi, W.Y.; Lautier, C.; Haydar, S.; Grigorescu, F.; Mahjoub, T. Association of estrogen receptor gene variants (ESR1 and ESR2) with polycystic ovary syndrome in Tunisia. Gene 2020, $741,144560$. [CrossRef]

12. Sundarrajan, C.; Liao, W.X.; Roy, A.C.; Ng, S.C. Association between Estrogen Receptor- $\beta$ Gene Polymorphisms and Ovulatory Dysfunctions in Patients with Menstrual Disorders. J. Clin. Endocrinol. Metab. 2001, 86, 135-139. [CrossRef] [PubMed]

13. Rani, A.; Roy, A. Variation in the ER $\alpha$ and ER $\beta$ Genes and Genetic Susceptibility to Polycystic Ovary Syndrome. Fertil. Steril. 2005, 84, S443. [CrossRef]

14. Valkenburg, O.; Uitterlinden, A.G.; Themmen, A.P.; de Jong, F.H.; Hofman, A.; Fauser, B.C.J.M.; Laven, J.S.E. Genetic polymorphisms of the glucocorticoid receptor may affect the phenotype of women with anovulatory polycystic ovary syndrome. Hum. Reprod. 2011, 26, 2902-2911. [CrossRef] [PubMed]

15. Nectaria, X.; Leandros, L.; Ioannis, G.; Agathocles, T. The importance of ER $\alpha$ and ER $\beta$ gene polymorphisms in PCOS. Gynecol. Endocrinol. 2012, 28, 505-508. [CrossRef]

16. Kim, J.J.; Choi, Y.M.; Choung, S.H.; Yoon, S.H.; Lee, G.H.; Moon, S.Y. Estrogen receptor beta gene +1730 G/A polymorphism in women with polycystic ovary syndrome. Fertil. Steril. 2010, 93, 1942-1947. [CrossRef]

17. Liaqat, I.; Jahan, N.; Krikun, G.; Taylor, H.S. Genetic Polymorphisms in Pakistani Women with Polycystic Ovary Syndrome. Reprod. Sci. 2014, 22, 347-357. [CrossRef] [PubMed]

18. Silva, F.; Sóter, M.; Sales, M.; Candido, A.; Reis, F.; Silva, I.; Sousa, M.; Ferreira, C.; Gomes, K. Estrogen receptor $\alpha$ lpha gene (ESR1) PvuII and XbaI polymorphisms are associated to metabolic and proinflammatory factors in polycystic ovary syndrome. Gene 2015, 560, 44-49. [CrossRef]

19. Qiao, J.; Han, B. Diseases caused by mutations in luteinizing hormone/chorionic gonadotropin receptor. Prog. Mol. Biol. Transl. Sci. 2019, 161, 69-89.

20. Owens, L.A.; Kristensen, S.G.; Lerner, A.; Christopoulos, G.; Lavery, S.; Hanyaloglu, A.C.; Hardy, K.; Andersen, C.Y.; Franks, S. Gene Expression in Granulosa Cells from Small Antral Follicles from Women with or without Polycystic Ovaries. J. Clin. Endocrinol. Metab. 2019, 104, 6182-6192. [CrossRef]

21. Valkenburg, O.; Uitterlinden, A.; Piersma, D.; Hofman, A.; Themmen, A.; de Jong, F.; Fauser, B.; Laven, J. Genetic polymorphisms of GnRH and gonadotrophic hormone receptors affect the phenotype of polycystic ovary syndrome. Hum. Reprod. 2009, 24, 2014-2022. [CrossRef]

22. Zou, J.; Wu, D.; Liu, Y.; Tan, S. Association of luteinizing hormone/choriogonadotropin receptor gene polymorphisms with polycystic ovary syndrome risk: A meta-analysis. Gynecol. Endocrinol. 2018, 35, 81-85. [CrossRef]

23. Thathapudi, S.; Kodati, V.; Erukkambattu, J.; Addepally, U.; Qurratulain, H. Association of Luteinizing Hormone Chorionic Gonadotropin Receptor Gene Polymorphism (rs2293275) with Polycystic Ovarian Syndrome. Genet. Test. Mol. Biomark. 2015, 19, 128-132. [CrossRef]

24. El-Shal, A.S.; Zidan, H.E.; Rashad, N.M.; Abdelaziz, A.M.; Harira, M.M. Association between genes encoding components of the Leutinizing hormone/Luteinizing hormone-choriogonadotrophin receptor pathway and polycystic ovary syndrome in Egyptian women. IUBMB Life 2016, 68, 23-36. [CrossRef]

25. Capalbo, A.; Sagnella, F.; Apa, R.; Fulghesu, A.M.; Lanzone, A.; Morciano, A.; Farcomeni, A.; Gangale, M.F.; Moro, F.; Martinez, D.; et al. The $312 \mathrm{~N}$ variant of the luteinizing hormone/choriogonadotropin receptor gene (LHCGR) confers up to $2 \cdot 7$-fold increased risk of polycystic ovary syndrome in a Sardinian population. Clin. Endocrinol. 2012, 77, 113-119. [CrossRef]

26. Mohiyiddeen, L.; Salim, S.; Mulugeta, B.; McBurney, H.; Newman, W.G.; Pemberton, P.; Nardo, L.G. PCOS and peripheral AMH levels in relation to FSH receptor gene single nucleotide polymorphisms. Gynecol. Endocrinol. 2012, 28, 375-377. [CrossRef] [PubMed] 
27. Laven, J.S.; Mulders, A.G.; Suryandari, D.A.; Gromoll, J.; Nieschlag, E.; Fauser, B.C.; Simoni, M. Follicle-stimulating hormone receptor polymorphisms in women with normogonadotropic anovulatory infertility. Fertil. Steril. 2003, 80, 986-992. [CrossRef]

28. Dolfin, E.; Guani, B.; Lussiana, C.; Mari, C.; Restagno, G.; Revelli, A. FSH-receptor Ala307Thr polymorphism is associated to polycystic ovary syndrome and to a higher responsiveness to exogenous FSH in Italian women. J. Assist. Reprod. Genet. 2011, 28, 925-930. [CrossRef] [PubMed]

29. Qiu, L.; Liu, J.; Hei, Q.-M. Association between Two Polymorphisms of Follicle Stimulating Hormone Receptor Gene and Susceptibility to Polycystic Ovary Syndrome: A Meta-analysis. Chin. Med. Sci. J. 2015, 30, 44-50. [CrossRef]

30. Wu, X.-Q.; Xu, S.-M.; Liu, J.-F.; Bi, X.-Y.; Wu, Y.-X.; Liu, J. Association between FSHR polymorphisms and polycystic ovary syndrome among Chinese women in north China. J. Assist. Reprod. Genet. 2014, 31, 371-377. [CrossRef] [PubMed]

31. Shi, Y.; Zhao, H.; Shi, Y.; Cao, Y.; Yang, D.; Li, Z.; Zhang, B.; Liang, X.; Li, T.; Chen, J.; et al. Genome-wide association study identifies eight new risk loci for polycystic ovary syndrome. Nat. Genet. 2012, 44, 1020-1025. [CrossRef]

32. Louwers, Y.; Stolk, L.; Uitterlinden, A.; Laven, J. Cross-Ethnic Meta-Analysis of Genetic Variants for Polycystic Ovary Syndrome. J. Clin. Endocrinol. Metab. 2013, 98, E2006-E2012. [CrossRef] [PubMed]

33. Zhao, S.; Tian, Y.; Gao, X.; Zhang, X.; Liu, H.; You, L.; Cao, Y.; Su, S.; Chan, W.-Y.; Sun, Y.; et al. Family-based analysis of eight susceptibility loci in polycystic ovary syndrome. Sci. Rep. 2015, 5, 12619. [CrossRef] [PubMed]

34. Uniprot. UniProtKB-P30968 (GNRHR_HUMAN). Available online: https:/ / www.uniprot.org/uniprot/P30968 (accessed on 1 January 2020).

35. Coutinho, E.A.; Kauffman, A.S. The Role of the Brain in the Pathogenesis and Physiology of Polycystic Ovary Syndrome (PCOS). Med. Sci. 2019, 7, 84. [CrossRef] [PubMed]

36. Li, Q.; Yang, G.; Wang, Y.; Zhang, X.; Sang, Q.; Wang, H.; Zhao, X.; Xing, Q.; He, L.; Wang, L. Common genetic variation in the $3^{\prime}$-untranslated region of gonadotropin-releasing hormone receptor regulates gene expression in cella and is associated with thyroid function, insulin secretion as well as insulin sensitivity in polycystic ovary syndrome patients. Qual. Life Res. 2011, 129, 553-561. [CrossRef]

37. Caburet, S.; Fruchter, R.B.; Legois, B.; Fellous, M.; Shalev, S.; Veitia, R.A. A homozygous mutation of GNRHR in a familial case diagnosed with polycystic ovary syndrome. Eur. J. Endocrinol. 2017, 176, K9-K14. [CrossRef] [PubMed]

38. Chen, W.-Y.; Du, Y.-Q.; Guan, X.; Zhang, H.-Y.; Liu, T. Effect of GnRHR polymorphisms on in vitro fertilization and embryo transfer in patients with polycystic ovary syndrome. J. Hum. Genet. 2017, 62, 1065-1071. [CrossRef] [PubMed]

39. Kowalczyk, K.; Franik, G.; Kowalczyk, D.; Pluta, D.; Blukacz, Ł.; Madej, P. Thyroid disorders in polycystic ovary syndrome. Eur. Rev. Med. Pharmacol. Sci. 2017, 21, 346-360.

40. Zawadzki, J.K. Diagnostic Criteria for Polycystic Ovary Syndrome: Towards a Rationale Approach. Polycystic Ovary Syndrome; Blackwell Scientific: Boston, MA, USA, 1992; pp. 39-50.

41. Eshre, T.R. Revised 2003 Consensus on Diagnostic Criteria and Long-Term Health Risks Related to Polycystic Ovary Syndrome. Fertil. Steril. 2004, 81, 19-25. 